\title{
Case Report \\ Renal Abscess in a Patient Presenting with Persistent Hiccups
}

\author{
Mark Flanagan, Katie Jennings, and Diann Krywko \\ Division of Emergency Medicine, Medical University of South Carolina, 169 Ashley Avenue, MSC 300, Room 294, \\ Charleston, SC 29425, USA \\ Correspondence should be addressed to Katie Jennings; jennink@musc.edu
}

Received 30 November 2012; Accepted 23 December 2012

Academic Editors: A. K. Exadaktylos, E. Kagawa, and F. Natale

Copyright (C) 2013 Mark Flanagan et al. This is an open access article distributed under the Creative Commons Attribution License, which permits unrestricted use, distribution, and reproduction in any medium, provided the original work is properly cited.

Hiccups are common, typically limited, and rarely present with adverse complications. In the context of persistent or intractable episodes, however, hiccups may signal a more serious underlying cause. Here, we present an unexpected and pathologic case of hiccups in a patient who was ultimately diagnosed with renal abscesses.

\section{Introduction}

Hiccups are also known as "hiccoughs", synchronous diaphragmatic flutter (SDF), and "singultus", from the Latin word singult, which roughly translates as "catch one's breath while sobbing" [1, 2]. Hiccups have usually been characterized as a benign annoyance and in most cases this proves true. However, persistent or intractable episodes are reported to have an organic cause approximately $80 \%$ of the time with the remaining $20 \%$ thought to be psychogenic in nature. Therefore, it is important to maintain a high index of suspicion in those patients to search for an underlying disease process.

\section{Case Report}

A 47-year-old male presented to the emergency department (ED) complaining of nausea, vomiting, diarrhea, stomach pain, and constant hiccups for three days. He had attributed his symptoms to having the flu and had been self-medicating with DayQuil and NyQuil without relief. The nausea and vomiting were not associated with eating or drinking and emesis was described as nonbloody and nonbilious. His past medical history was significant for previously treated tuberculosis. He denied any medications, allergies, or past surgeries. He lived alone and denied any current use of alcohol, tobacco, or illicit drug use but endorsed prior abuse of alcohol, cocaine, and marijuana. He was an unemployed construction worker with no recent travel.
Review of systems revealed increased fatigue over the prior 4-5 days, an estimated 30 pound unexpected weight loss over the past three months, peeling of palms and recent upper respiratory infection symptoms, but was otherwise negative.

Upon arrival, his vital signs were as follows: oral temperature $97.3^{\circ} \mathrm{F}$, blood pressure $123 / 78$, heart rate 72 , respiration rate 20 , and an oxygen saturation of $96 \%$ on room air. He was noted to be persistently hiccupping, although in no acute distress and generally well appearing. Examination of the ENT, neck, chest, and cardiovascular system were within normal limits. Abdominal exam was soft and exhibited normal bowel sounds. There was mild epigastric tenderness to palpation, no guarding, no rebound, and no masses. Rectal exam revealed guaiac positive stool. He was noted to have mild desquamation of his palms bilaterally.

ED laboratory results were significant for WBC $28 \mathrm{~K} /$ cumm, hemoglobin $12 \mathrm{gm} / \mathrm{dL}$, hematocrit $32 \%$, and platelets of $84 \mathrm{~K} /$ cumm. Sodium was $125 \mathrm{mmol} / \mathrm{L}$, bicarbonate $20 \mathrm{mmol} / \mathrm{L}$, BUN $121 \mathrm{mg} / \mathrm{dL}$, and creatinine $5.3 \mathrm{mg} / \mathrm{dL}$. An ED visit 4 years prior demonstrated a creatinine of $1.0 \mathrm{mg} / \mathrm{dL}$. Bilirubin was $4.9 \mathrm{mg} / \mathrm{dL}$, AST $185 \mathrm{IU} / \mathrm{L}$, ALT $93 \mathrm{IU} / \mathrm{L}$, and ALP $158 \mathrm{IU} / \mathrm{L}$. The urinalysis revealed 11-20/HPF WBCs, positive nitrates, and many bacteria but was otherwise negative. A KUB X-ray was obtained demonstrating a $1.2 \times$ $0.7 \mathrm{~cm}$ rounded opacity overlaying the expected course of the left ureter.

The patient was administered normal saline IV fluids and chlorpromazine $25 \mathrm{mg}$ IV with temporizing affect of the hiccups. A workup for postobstructive uropathy was 


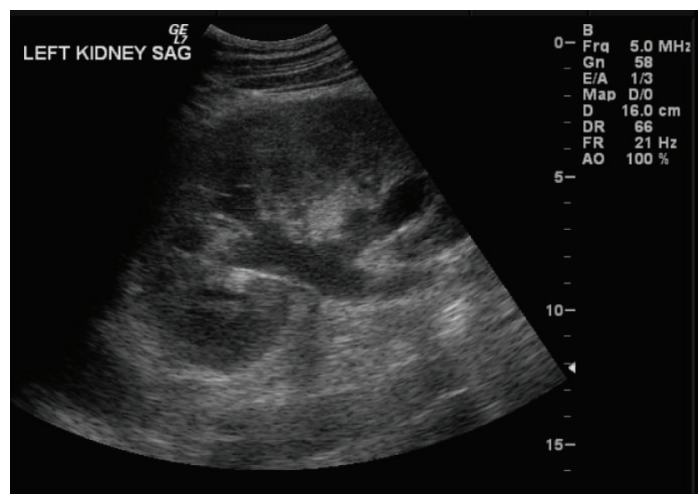

Figure 1: Renal ultrasound demonstrating mild left hydronephrosis.

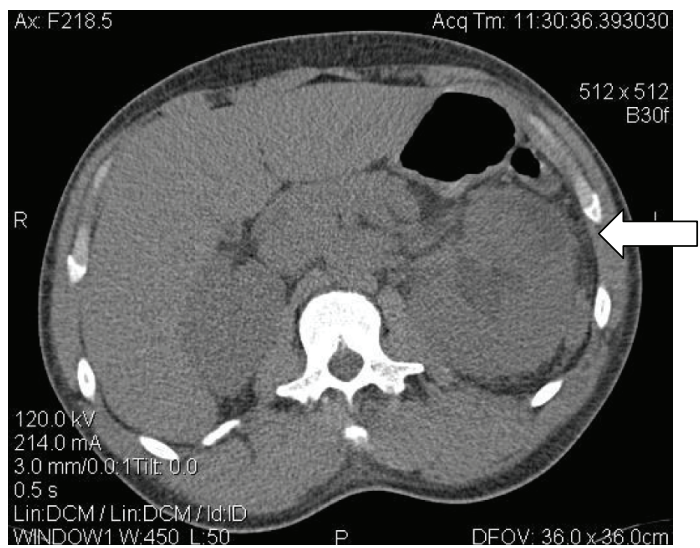

FIGURE 2: Enlarged left kidney with a ventral superior hypodensity concerning for a subcapsular fluid collection.

initiated, including a renal ultrasound which showed mild left hydronephrosis with a proximal $1.1 \mathrm{~cm}$ ureteral stone (Figure 1).

Urology was consulted and a CT IVP was obtained. In addition to prior findings of hydronephrosis and a $1.1 \mathrm{~cm}$ ureteral stone, a subcapsular fluid collection concerning for an abscess was noted overlying the left kidney (Figure 2). The patient was started on empiric IV ciprofloxacin and admitted to the Internal Medicine service. An emergent percutaneous nephrostomy tube was placed.

During the patient's hospital admission, he was converted to an oral antibiotic regimen which initially responded well to therapy. It was noted, however, that the patient continued to experience persistent hiccups. A subsequent increased leukocytosis and recurrent fever prompted a repeat CT IVP which demonstrated another left subcapsular fluid collection concerning for abscess. A second percutaneous nephrostomy tube was placed and an additional course of IV antibiotics started, with subsequent resolution of the patient's hiccups and normalization of his renal function. He was discharged in good condition 3 days later and subsequently lost to followup.

\section{Discussion}

Hiccups are not known to serve any physiological purpose and are known to start in vitro. Episodes of hiccups are typically brief and may be bothersome but are rarely of serious consequence. As episodes lengthen and increase in frequency, they may signal a more serious underlying condition. Persistent hiccups are defined as those lasting greater than 48 hours whereas intractable hiccups are defined as lasting more than 1 month. Persistent and intractable hiccups occur more frequently with the adult, male population for as yet unrecognizable reasons [1-3].

The pathophysiology of hiccups remains somewhat poorly understood. There is general consensus that they are a reflex pattern of "sudden contraction of the diaphragmatic inspiratory muscles followed by an abrupt closure of the glottis" $[1,2]$. The hiccup reflex arc is comprised of an afferent limb emanating from the phrenic and vagus nerves and the sympathetic chain from T6 to T12. It then passes through a "hiccup center" located in a nonspecific region of the C3 to C5 cervical segments. An efferent limb of the hiccup arc routes primarily through the phrenic nerve, affecting the diaphragm and causing muscular contraction, though recent studies $[2,3]$ ascribe there may be several different as yet identified components of the efferent limb. Fluoroscopy has shown that hiccups typically affect only one hemidiaphragm with the left hemi-diaphragm being affected about $80 \%$ of the time.

There are many causes of hiccups. The etiology of benign or self-limited hiccups is often attributed to gastric distention, sudden changes in ambient or gastric temperature, consumption of alcohol, or smoking tobacco [1]. The pathologic etiologies can be subdivided into central or peripheral processes, toxic or metabolic processes, or pharmacologic processes.

Central causes of hiccups can be structural, vascular, or infectious in nature. Etiologies include, but are not limited to: central neoplasms, multiple sclerosis, cerebrovascular accidents, arteriovenous malformations, encephalitis, and meningitis [1-3].

Peripheral causes are far vaguer and numerous in nature, including any process which can irritate the vagus or phrenic nerves and its branches. These include, but are not limited to, pharyngitis, retropharyngeal, or paratonsillar abscess, goiters, tumors of the neck or mediastinum, irritation of the tympanic membranes, myocardial infarction, pericarditis, and aortic aneurysm, or dissection. Any process that causes pulmonary or intra-abdominal irritation, which can then precipitate irritation of the diaphragm, must be included in this very long list of differential diagnoses. It should also be noted that foreign bodies, such as aberrant pacemaker lines, may cause diaphragmatic irritation.

Toxic and metabolic causes include alcohol ingestion, uremia, hyponatremia, and hypocalcaemia. Some drugs are also known to lead to hiccups, prompting a thorough evaluation of the patient's medication list. These pharmacologic agents include IV steroids, barbiturates, benzodiazepines, and alpha-methyldopa. 
Management of hiccups should center on a thorough history and physical as well as the inclusion of pertinent laboratory and radiographic studies in order to rule out an organic cause if suspected. Treatment of an organic cause of hiccups usually resolves the hiccups. There are a large percentage of patients for whom no etiology can be found. For those, there are a multitude of potential nonpharmacologic and pharmacologic treatments.

Few nonpharmacologic treatments have been medically well-studied but may be worth attempting for shorter bouts, if no contraindication exists. Two common techniques include breath holding or bag breathing. Both serve to increase arterial $\mathrm{pCO}_{2}$. This may decrease the hiccup frequency but will not stop them completely. Irritation of the nasopharynx is thought to interrupt the hiccup reflex arc. This may be accomplished by direct means through nasopharyngeal suction or uvular stimulation, or by more indirect means of swallowing a spoonful of granulated sugar or gargling with cold water [2]. Other methods such as acupuncture and hypnosis have been attempted with reported success [1-5]. More severe cases have also been treated with phrenic nerve blockade or surgical interruption [1].

Pharmacological treatments for the management of hiccups continues to evolve. Some of the more common drugs utilized include nifedipine, haloperidol, phenytoin, metoclopramide, and gabapentin. The two most commonly utilized medications are chlorpromazine and baclofen. Chlorpromazine is the most widely used medication for persistent hiccups [6]. The recommended starting dose is $25-50 \mathrm{mg}$ IV. It may also be administered orally or intramuscularly but with reduced effectiveness. This dose may be repeated up to three times. If successful, chlorpromazine may be continued orally as an outpatient at a dose of $25-50 \mathrm{mg}$ up to 3-4 times a day for 10 days [7]. Baclofen also has been demonstrated to be effective in moderating or terminating persistent hiccups with no demonstrable organic cause. Its mechanism of action in terminating hiccups is unclear, but presumably central [8]. Dosing may be done in conjunction with famotidine when a gastrointestinal cause is suspected [9]. Recommended initial dosing is $10 \mathrm{mg}$ orally, three times a day [10].

\section{Summary}

The evaluation of a patient with the chief complaint of persistent or intractable hiccups can be a daunting process given the broad list of differential diagnoses to consider. Though many patients will leave the ED with the diagnosis of idiopathic singultus, it is vital to maintain a high index of suspicion in order to rule out a potentially life threatening etiology.

\section{Disclosure}

The authors of this paper have no financial disclosure or conflict of interests to resolve.

\section{References}

[1] P. W. Kolodzik and M. A. Eilers, "Hiccups (singultus): review and approach to management," Annals of Emergency Medicine, vol. 20, no. 5, pp. 565-573, 1991.
[2] J. H. Lewis, "Hiccups: causes and cures," Journal of Clinical Gastroenterology, vol. 7, no. 6, pp. 539-552, 1985.

[3] R. PauL, "Hiccups," Southern Medical Journal, vol. 88, no. 2, pp. 175-181, 1995.

[4] J. Dietzel, M. Grundling, D. Pavlovic, and T. I. Usichenko, "Acupuncture for persistent postoperative hiccup," Anaesthesia, vol. 63, no. 9, pp. 1021-1022, 2008.

[5] M. Bobele, "Nonmedical management of intractible hiccups: a brief review of the literature," Psychological Reports, vol. 61, no. 1, pp. 225-226, 1987.

[6] I. Gilson and M. Busalacchi, "Marijuana for intractable hiccups," The Lancet, vol. 351, no. 9098, p. 267, 1998.

[7] J. E. Tintinalli, D. Gabor, and J. Stephan, Stapczynski, Emergency Medicine: A Comprehensive Study Guide, 6th edition, 2004.

[8] K. Marsot-Dupuch, V. Bousson, J. Cabane, and J. M. Tubiana, "Intractable hiccups: the role of cerebral MR in cases without systemic cause," American Journal of Neuroradiology, vol. 16, no. 10, pp. 2093-2100, 1995.

[9] C. Guelaud, T. Similowski, J. L. Bizec, J. Cabane, W. A. Whitelaw, and J. P. Derenne, "Baclofen therapy for chronic hiccup," European Respiratory Journal, vol. 8, no. 2, pp. 235-237, 1995.

[10] K. Marien and D. Havlak, "Baclofen with famotidine for intractable hiccups," European Respiratory Journal, vol. 10, no. 9, p. 2188, 1997. 


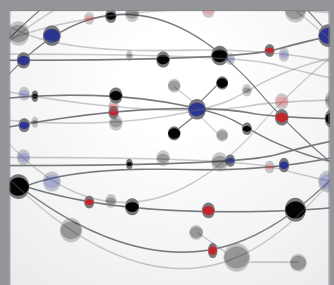

The Scientific World Journal
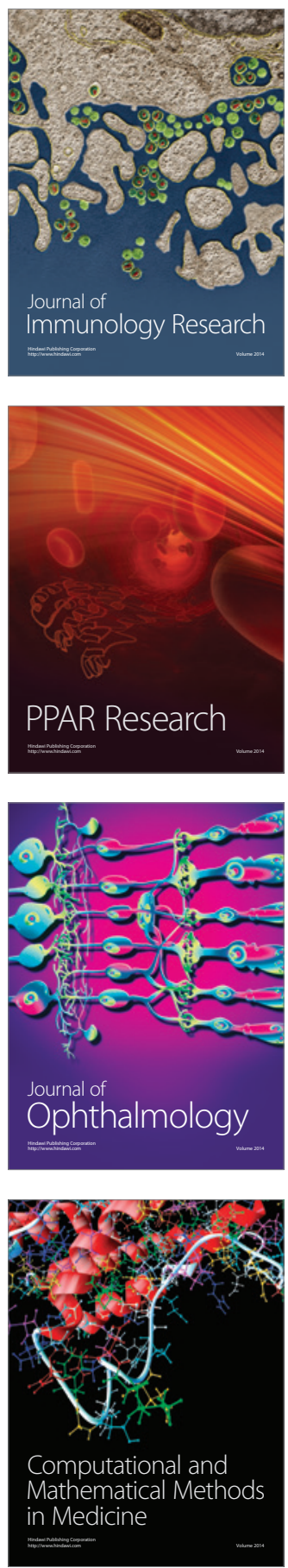

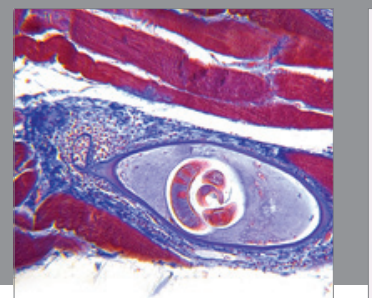

Gastroenterology

Research and Practice
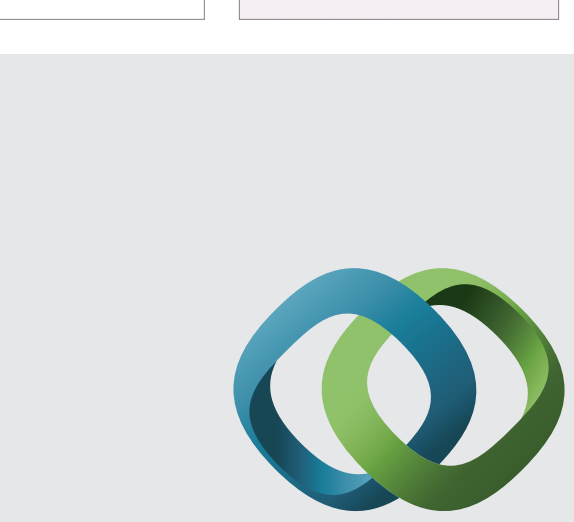

\section{Hindawi}

Submit your manuscripts at

http://www.hindawi.com
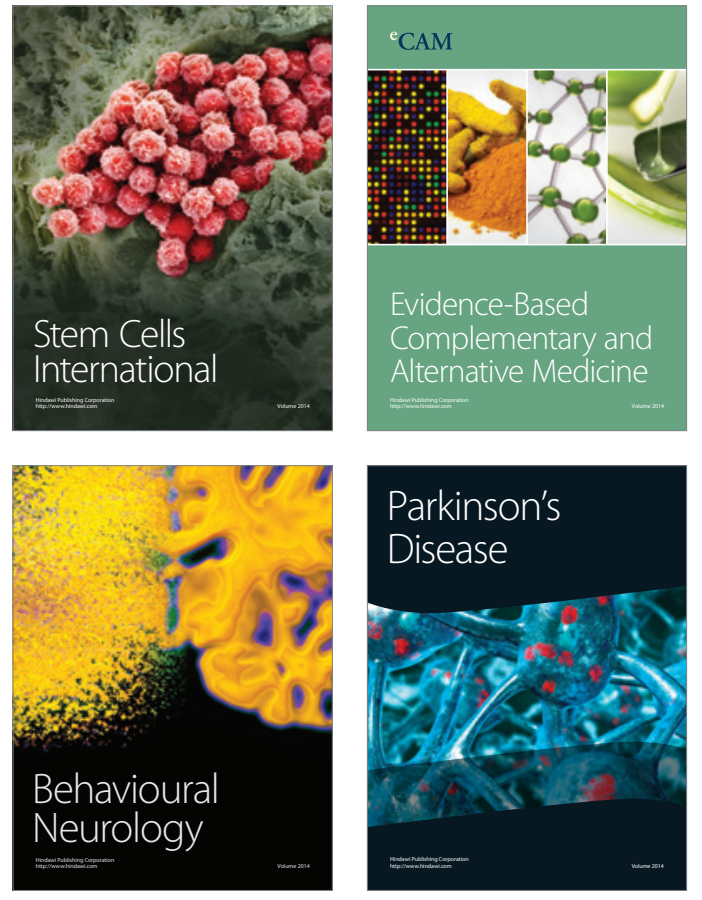
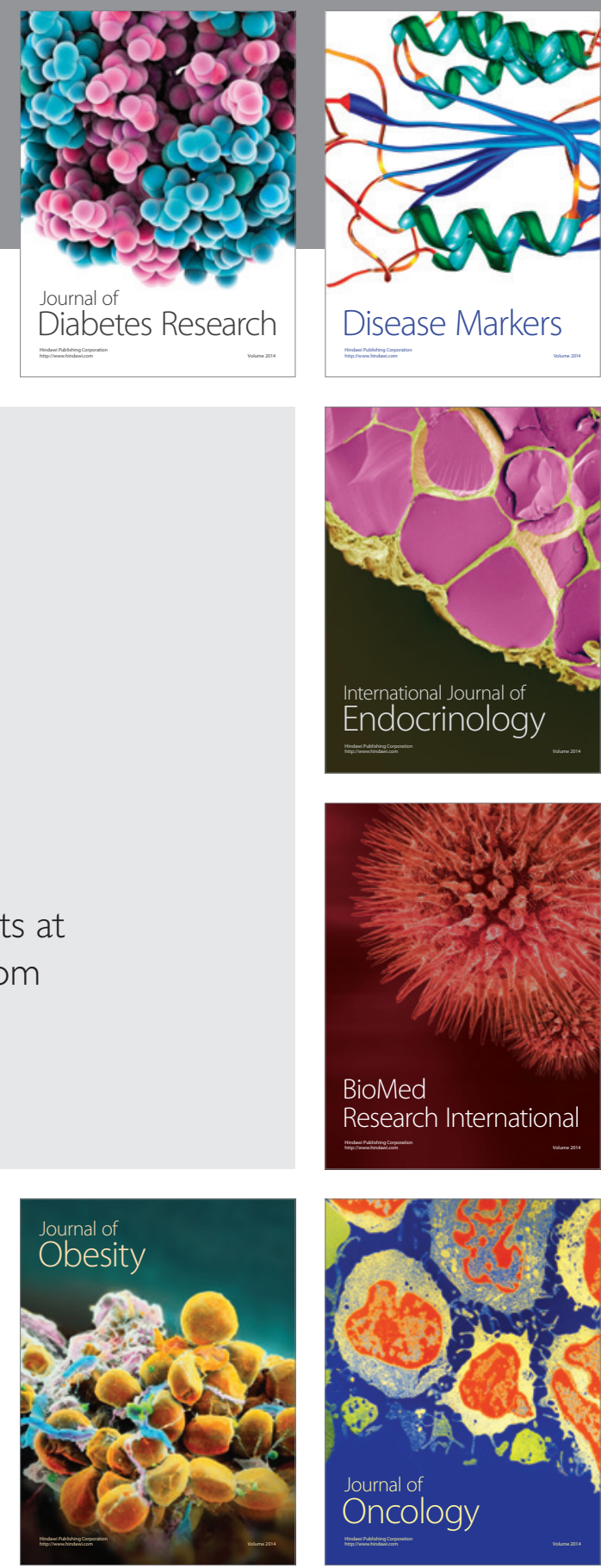

Disease Markers
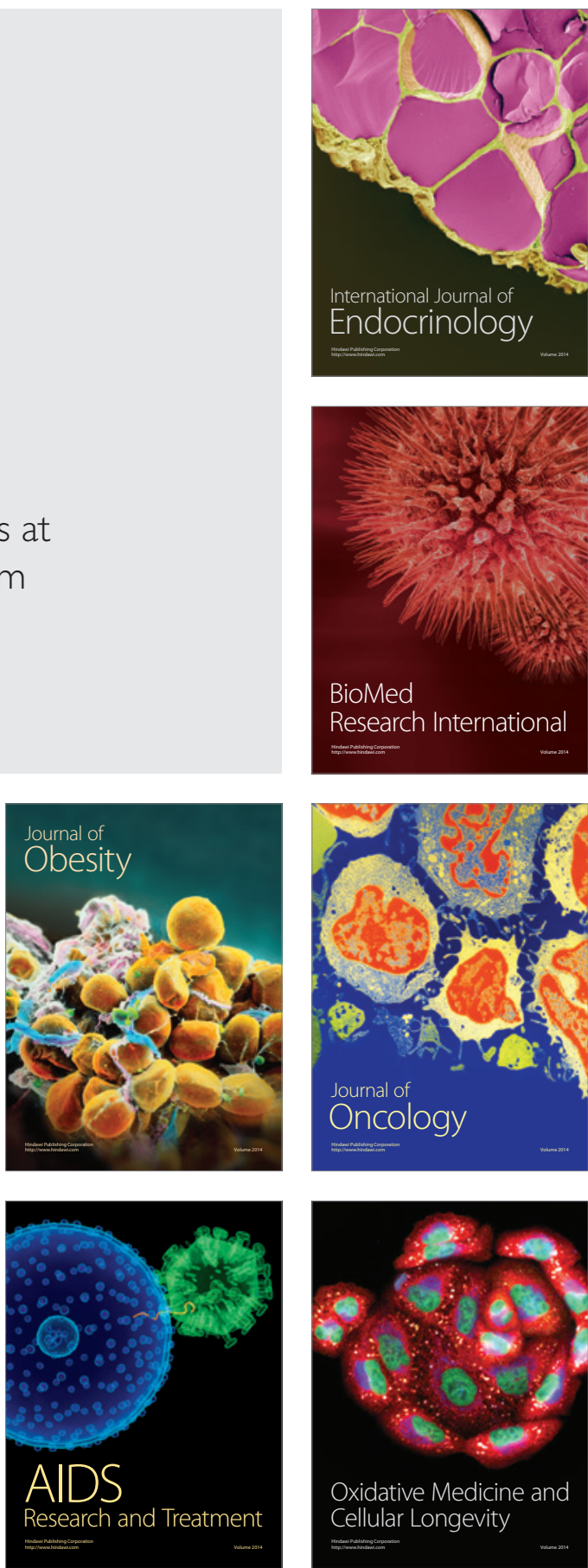